\title{
A Note on the Global Attractivity of a Discrete Model of Nicholson's Blowflies*
}

\author{
B.G. ZHANG ${ }^{\dagger}$ and H.X. XU \\ Department of Applied Mathematics, Ocean University of Qingdao, Qingdao 266003, P.R. China \\ (Received 4 February 1999)
}

In this paper, we further study the global attractivity of the positive equilibrium of the discrete Nicholson's blowflies model

$$
N_{n+1}-N_{n}=-\delta N_{n}+p N_{n-k} \mathrm{e}^{-a N_{n-k}}, \quad n=0,1,2, \ldots
$$

We obtain a new criterion for the positive equilibrium $N^{*}$ to be a global attractor, which improve the corresponding results obtained by So and Yu (J. Math. Anal. Appl. 193 (1995), 233-244).

Keywords: Attractivity, Positive equilibrium, Discrete Nicholson's blowflies model

AMS Subject Classification: 39A10

\section{INTRODUCTION}

The delay difference equation

$$
\begin{aligned}
& N_{n+1}-N_{n}=-\delta N_{n}+p N_{n-k} \mathrm{e}^{-a N_{n-k}}, \\
& \quad n=0,1,2, \ldots,
\end{aligned}
$$

is a discrete analogue of the delay differential equation

$$
N^{\prime}(t)=-\delta N(t)+p N(t-\tau) \mathrm{e}^{-a N(t-\tau)}, \quad t \geq 0,
$$

which has been used in describing the dynamics of Nicholson's blowflies $[2,4-6]$.

* This work is supported by NNSF of China.

${ }^{\dagger}$ Corresponding author.
By the biology consideration, we assume that $\delta \in(0,1), p, a \in(0,+\infty)$, and $k \in N=\{0,1,2, \ldots\}$. The initial condition is

$$
N_{j}=\varphi_{j} \geq 0, \quad j \in\{-k,-k+1, \ldots, 0\},
$$

and $\varphi_{j}>0$, for some $j \in\{-k,-k+1, \ldots, 0\}$.

By a solution of (1) and (2) we mean a sequence $\left\{N_{n}\right\}$ which satisfies (1) for $n=0,1,2, \ldots$ as well as the initial condition (2). Clearly, the unique solution $\left\{N_{n}\right\}$ of the above initial value problem is positive for all large $n$ [1]. 
If $p>\delta$, then Eq. (1) has a unique positive equilibrium $N^{*}$ and

$$
N^{*}=\frac{1}{a} \ln \left(\frac{p}{\delta}\right) .
$$

The global attractivity of $N^{*}$ was studied by Kocic and Lada [3] and So and $\mathrm{Yu}$ [1] respectively.

The recent result is the following [1].

TheOrem A Assume that $p>\delta$ and that

$$
\left[(1-\delta)^{-k-1}-1\right] \ln \left(\frac{p}{\delta}\right) \leq 1
$$

Then any nontrival solution $N_{n}$ of (1) and (2) satisfies

$$
\lim _{n \rightarrow \infty} N_{n}=N^{*}
$$

In this note, our purpose is to improve condition (4). Exactly speaking, we will show some conditions for the global attractivity of $N^{*}$ when (4) does not hold. Our results are discrete analogues of the results in [2].

To prove our main results, we need some known results.

LEMMA 1 [1] Let $\left\{N_{n}\right\}$ be a solution of (1) and (2). Then

$$
\limsup _{n \rightarrow \infty} N_{n} \leq \frac{p}{a e \delta} .
$$

As in [2], the following system of inequalities

$$
\left\{\begin{array}{l}
y+\ln \left(1+\left(y / a N^{*}\right)\right) \leq M\left(\mathrm{e}^{-x}-1\right) \\
x+\ln \left(1+\left(x / a N^{*}\right)\right) \geq M\left(\mathrm{e}^{-y}-1\right)
\end{array}\right.
$$

play an important role in our analysis, where $M=$ $a N^{*}\left[(1-\delta)^{-k-1}-1\right]=\left[(1-\delta)^{-k-1}-1\right] \ln (p / \delta)$.

Let

$$
D=\left\{(x, y):-a N^{*}<x \leq 0 \leq y<\infty\right\} .
$$

LEMMA 2 [2] If one of the following conditions holds:

(i) $M \leq 1$;

(ii) $M<1+\left(1 / a N^{*}\right)$ and $a N^{*} \geq(\sqrt{5}-1) / 2$; (iii) $M \leq 1+\left(1 / a N^{*}\right)$ and

$$
a N^{*}>(\sqrt{1+4 \sqrt{3}}-1) / 2 \text {, }
$$

then (6) has a unique solution $x=y=0$ in $D$.

\section{MAIN RESULTS}

The following theorem provides a new sufficient condition for the equilibrium $N^{*}=(1 / a) \ln (p / \delta)$ to be a global attractor.

THEOREM 1 Assume that $p>\delta$ and the assumption in Lemma 2 holds. Then any nontrivial solution $\left\{N_{n}\right\}$ of (1) and (2) satisfies

$$
\lim _{n \rightarrow \infty} N_{n}=N^{*}
$$

Proof Let

$$
N_{n}=N^{*}+\frac{1}{a} x_{n}
$$

Then $\left\{x_{n}\right\}$ is a solution of the equation

$$
\begin{aligned}
& x_{n+1}-x_{n}+\delta x_{n}+a \delta N^{*}\left(1-\mathrm{e}^{-x_{n-k}}\right) \\
& \quad-\delta x_{n-k} \mathrm{e}^{-x_{n-k}}=0, \quad n=0,1,2, \ldots
\end{aligned}
$$

Since $N_{n}>0$ for all large $n$, it follows that $x_{n}>-a N^{*}$ for all large $n$.

To prove this theorem, it is sufficient to prove $\lim _{n \rightarrow \infty} x_{n}=0$. Lemma 1 implies that $\left\{x_{n}\right\}$ is bounded above. Let

$$
\mu=\limsup _{n \rightarrow \infty} x_{n} \text { and } \lambda=\liminf _{n \rightarrow \infty} x_{n} .
$$

Then $-a N^{*} \leq \lambda \leq \mu<\infty$. We claim that $\lambda=\mu=0$. For the case $\left\{x_{n}\right\}$ is eventually nonnegative or eventually nonpositive, this has been proved in the proof of Theorem 2 in [3]. Therefore it is sufficient to consider the case that $\left\{x_{n}\right\}$ is an oscillatory solution of (8).

Our purpose is to prove that $\lambda=\mu=0$ under the assumptions. There are four possible cases:

(1) $\lambda=\mu=0$;

(2) $\mu>0$ and $\lambda=0$; 
(3) $\mu=0$ and $\lambda<0$;

(4) $\mu>0$ and $\lambda<0$.

The cases 2 and 3 can be considered to be special cases of case 4 . Now we consider case 4 .

In this case, there exists a sequence $\left\{n_{i}\right\}$ of positive integers such that

$$
\begin{gathered}
k<n_{1}<n_{2}<\cdots<n_{i}<n_{i+1} \rightarrow \infty \quad \text { as } i \rightarrow \infty . \\
x_{n_{i}}<0 \text { and } x_{n_{i}+1} \geq 0, \quad \text { for } i=1,2, \ldots,
\end{gathered}
$$

and for each $i=1,2, \ldots$, the terms of the finite sequence $x_{j}$ for $n_{i}<j<n_{i+1}$ assume both positive and negative values. Let $m_{i}$ and $M_{i}$ be integers in $\left(n_{i}, n_{i+1}\right)$ such that for $i=1,2, \ldots$

$$
x_{M_{i}}=\max \left\{x_{j}: n_{i}<j<n_{i+1}\right\},
$$

and

$$
x_{m_{i}}=\min \left\{x_{j}: n_{i}<j<n_{i+1}\right\} .
$$

We can assume without loss of generality that for $i=1,2, \ldots$.

$$
\begin{gathered}
x_{M_{i}}>0, \quad x_{M_{i}}-x_{M_{i}-1} \geq 0 \quad \text { and } \\
\limsup _{i \rightarrow \infty} x_{M_{i}}=\mu>0,
\end{gathered}
$$

while

$$
\begin{gathered}
x_{m_{i}}<0, \quad x_{m_{i}}-x_{m_{i}-1} \leq 0 \quad \text { and } \\
\liminf _{i \rightarrow \infty} x_{m_{i}}=\lambda<0 .
\end{gathered}
$$

Then there exist subsequence $\left\{q_{i}\right\}$ of $\left\{m_{i}\right\}$ and subsequence $\left\{Q_{i}\right\}$ of $\left\{M_{i}\right\}$ such that

$$
\begin{aligned}
& x_{Q_{i}}>0, \quad x_{Q_{i}}-x_{Q_{i}-1} \geq 0 \quad \text { and } \\
& \lim _{i \rightarrow \infty} x_{Q_{i}}=\mu>0,
\end{aligned}
$$

while

$$
\begin{gathered}
x_{q_{i}}<0, \quad x_{q_{i}}-x_{q_{i}-1} \leq 0 \quad \text { and } \\
\lim _{i \rightarrow \infty} x_{q_{i}}=\lambda<0 .
\end{gathered}
$$

It follows from (8) and (10) that

$$
x_{Q_{i}-1}+a N^{*} \leq\left[x_{Q_{i}-k-1}+a N^{*}\right] \mathrm{e}^{-x_{Q_{i}-k-1}},
$$

thus

$$
\begin{aligned}
x_{Q_{i}}+a N^{*}= & (1-\delta)\left(x_{Q_{i}-1}+a N^{*}\right) \\
& +\delta\left(x_{Q_{i}-k-1}+a N^{*}\right) \mathrm{e}^{-x_{Q_{i}-k-1}} \\
\leq & (1-\delta)\left(x_{Q_{i}-k-1}+a N^{*}\right) \mathrm{e}^{-x_{Q_{i}-k-1}} \\
& +\delta\left(x_{Q_{i}-k-1}+a N^{*}\right) \mathrm{e}^{-x_{Q_{i}-k-1}} \\
= & \left(x_{Q_{i}-k-1}+a N^{*}\right) \mathrm{e}^{-x_{Q_{i}-k-1}}
\end{aligned}
$$

that is

$$
x_{Q_{i}}+a N^{*} \leq\left(x_{Q_{i}-k-1}+a N^{*}\right) \mathrm{e}^{-x_{Q^{-k-1}}} .
$$

Now let us prove

$$
x_{Q_{i}-k-1}<0,
$$

assume the contrary, then $x_{Q_{i}-k-1}=0$ or $x_{Q_{i}-k-1}>0$. If $x_{Q_{i}-k-1}=0$, then $x_{Q_{i}} \leq 0$, which contradicts (10). If $x_{Q_{i}-k-1}>0$, then $x_{Q_{i}-k-1}>x_{Q_{i}}$, thus

$$
\liminf _{i \rightarrow \infty} x_{Q_{i}-k-1} \geq \liminf _{i \rightarrow \infty} x_{Q_{i}}=\mu,
$$

on the other hand, we have

$$
\limsup _{i \rightarrow \infty} x_{Q_{i}-k-1} \leq \limsup _{i \rightarrow \infty} x_{M_{i}}=\mu,
$$

so we get

$$
\lim _{i \rightarrow \infty} x_{Q_{i}-k-1}=\mu,
$$

then taking the limit in (12), we obtain

$$
\mu+a N^{*} \leq\left(\mu+a N^{*}\right) \mathrm{e}^{-\mu},
$$

which implies $\mu \leq 0$ that contradicts (10), so (13) holds.

From (12) and (13), we have

$$
x_{Q_{i}}+a N^{*}<a N^{*} \mathrm{e}^{-x_{Q_{i}-k-1}},
$$


therefore

$$
x_{Q_{i}-k-1}<-\ln \left(1+\frac{x_{Q_{i}}}{a N^{*}}\right) .
$$

For given $\varepsilon>0$, by (9), there exists a positive integer $n^{*}$ such that

$$
\lambda-\varepsilon<x_{n}<\mu+\varepsilon, \quad \text { for } n \geq n^{*}-k,
$$

this induce $x_{n-k} \mathrm{e}^{-x_{n-k}}<\mu+\varepsilon$, for $n \geq n^{*}$.

Rewriting Eq. (8) into the following form:

$$
\begin{aligned}
& (1-\delta)^{-n-1} x_{n+1}-(1-\delta)^{-n} x_{n} \\
& +a \delta N^{*}(1-\delta)^{-n-1}\left(1-e^{-x_{n-k}}\right) \\
& \quad-\delta(1-\delta)^{-n-1} x_{n-k} \mathrm{e}^{-x_{n-k}}=0
\end{aligned}
$$

Now summing (16) up from $n=Q_{i}-k-1$ (assuming $Q_{i}-k-1 \geq n^{*}$ ) to $n=Q_{i}-1$. we have

$$
\begin{aligned}
(1-\delta)^{-Q_{i}} x_{Q_{i}}= & (1-\delta)^{-Q_{i}+k+1} x_{Q_{i}-k-1}-a \delta N^{*} \\
& \times \sum_{n=Q_{i}-k-1}^{Q_{i}-1}(1-\delta)^{-n-1}\left(1-\mathrm{e}^{-x_{n-k}}\right) \\
& +\delta \sum_{n=Q_{i}-k-1}^{Q_{i}-1}(1-\delta)^{-n-1} x_{n-k} \mathrm{e}^{-x_{n-k}} \\
< & (1-\delta)^{-Q_{i}+k+1} x_{Q_{i}-k-1}+a \delta N^{*} \\
& \times \sum_{n=Q_{i}-k-1}^{Q_{i}-1}(1-\delta)^{-n-1}\left(\mathrm{e}^{-\lambda+\varepsilon}-1\right) \\
& +\delta \sum_{n=Q_{i}-k-1}^{Q_{i}-1}(1-\delta)^{-n-1}(\mu+\varepsilon) \\
= & (1-\delta)^{-Q_{i}+k+1} x_{Q_{i}-k-1} \\
& +\left[(\mu+\varepsilon)+a N^{*}\left(\mathrm{e}^{-\lambda+\varepsilon}-1\right)\right] \\
& \times(1-\delta)^{-Q_{i}}\left[1-(1-\delta)^{k+1}\right] .
\end{aligned}
$$

Substituting (15) into the above inequality, we get

$$
\begin{aligned}
(1-\delta)^{-Q_{i}} x_{Q_{i}}< & -(1-\delta)^{-Q_{i}+k+1} \ln \left(1+\frac{x_{Q_{i}}}{a N^{*}}\right) \\
+ & {\left[(\mu+\varepsilon)+a N^{*}\left(\mathrm{e}^{-\lambda+\varepsilon}-1\right)\right] } \\
& \times(1-\delta)^{-Q_{i}}\left[1-(1-\delta)^{k+1}\right],
\end{aligned}
$$

and

$$
\begin{aligned}
x_{Q_{i}} & +(1-\delta)^{k+1} \ln \left(1+\frac{x_{Q_{i}}}{a N^{*}}\right) \\
& <\left[(\mu+\varepsilon)+a N^{*}\left(\mathrm{e}^{-\lambda+\varepsilon}-1\right)\right]\left[1-(1-\delta)^{k+1}\right],
\end{aligned}
$$

let $i \rightarrow \infty, \varepsilon \rightarrow 0$, we get

$$
\begin{aligned}
\mu+ & (1-\delta)^{k+1} \ln \left(1+\frac{\mu}{a N^{*}}\right) \\
& \leq\left[\mu+a N^{*}\left(\mathrm{e}^{-\lambda}-1\right)\right]\left[1-(1-\delta)^{k+1}\right] .
\end{aligned}
$$

We rewrite the above inequality:

$$
\mu+\ln \left(1+\frac{\mu}{a N^{*}}\right) \leq M\left(\mathrm{e}^{-\lambda}-1\right) .
$$

In a similar way, we have

$$
\lambda+\ln \left(1+\frac{\lambda}{a N^{*}}\right) \geq M\left(\mathrm{e}^{-\mu}-1\right) .
$$

Then we establish the following system of inequalities:

$$
\left\{\begin{array}{l}
\mu+\ln \left(1+\left(\mu / a N^{*}\right)\right) \leq M\left(\mathrm{e}^{-\lambda}-1\right), \\
\lambda+\ln \left(1+\left(\lambda / a N^{*}\right)\right) \geq M\left(\mathrm{e}^{-\mu}-1\right) .
\end{array}\right.
$$

For case 2, the system of inequalities corresponding to (19) is

$$
\left\{\begin{array}{l}
\mu+\ln \left(1+\left(\mu / a N^{*}\right)\right) \leq M\left(\mathrm{e}^{-\lambda}-1\right), \\
\lambda=0 .
\end{array}\right.
$$

It is obvious that (20) holds iff $\lambda=\mu=0$.

For case 3 , the system of inequalities corresponding to (19) is

$$
\left\{\begin{array}{l}
\mu=0, \\
\lambda+\ln \left(1+\left(\lambda / a N^{*}\right)\right) \geq M\left(\mathrm{e}^{-\mu}-1\right) .
\end{array}\right.
$$

Similarly, (21) holds iff $\lambda=\mu=0$.

Thus it will suffice to consider case 4 , for (19) in case 4 , by Lemma 2 , we get $\lambda=\mu=0$. So the proof is complete. 
Remark 1 In cases 2 and 3 in Theorem 1, we add some reasonable conditions to $a N^{*}$. We know

$$
M=a N^{*}\left[(1-\delta)^{-k-1}-1\right] \leq 1+\frac{1}{a N^{*}},
$$

on the right side of which there is nothing to do with $\delta$ and $k$. While $1+\left(1 / a N^{*}\right) \rightarrow \infty$ as $a N^{*} \rightarrow 0+$, properly choosing the values of $\left[(1-\delta)^{-k-1}-1\right]$, we can let $M$ equal or infinitely tend to the value of $1+\left(1 / a N^{*}\right)$, then $M$ can be changed to arbitrarily large. Obviously this is not reasonable.

Remark 2 Theorem 4.1 in [1] only applies to the case $M \leq 1$, while Theorem 1 in this paper not only applies to $M \leq 1$ but also to $M>1$. So the results in this paper improve those in [1].

Example Consider the delay difference equation

$$
N_{n+1}-N_{n}=-\frac{1}{4} N_{n}+\frac{1}{4} \mathrm{e}^{(\sqrt{5}-1) / 2} N_{n-3} \mathrm{e}^{-2 N_{n-3}},
$$

then we can calculate

$$
a N^{*}=\frac{\sqrt{5}-1}{2} \text { and }\left[(1-\delta)^{-k-1}-1\right]=\frac{175}{81}
$$

thus,

$$
M \approx 1.335 \text { and } 1+\frac{1}{a N^{*}}=\frac{\sqrt{5}+3}{2} \approx 2.618
$$

The conditions in Theorem 1 are satisfied. Thus

$$
N^{*}=\frac{\sqrt{5}-1}{4}
$$

is a global attractor or (22). But Theorem 4.1 in [1] cannot apply to this case.

\section{References}

[1] J.W.-H. So and J.S. Yu. On the stability and uniform persistence of a discrete model of Nicholson's blowflies. J. Math. Anal. Appl. 193 (1995), 233-244.

[2] Li Jingwen. Global attractivity in Nicholson's blowflies, Appl. Math.-JCU 11B (1996), 425-436.

[3] V.Lj. Kocic and G. Ladas. Oscillation and attractivity in a discrete model of Nicholson's blowflies, Appl. Anal. 38 (1990), 21-31.

[4] W.S. Gurney, S.P. Blythe and R.M. Nisbet. Nicholson's blowflies revisited. Nature 287 (1980), 17-21.

[5] M.R.S. Kulenovic, G. Ladas and Y.G. Sficas. Global attractivity in Nicholson's blowflies, Appl. Anal. 43 (1992), $109-124$.

[6] J.W.-H. So and J.S. Yu. Global attractivity and uniformly persistence in Nicholson's blowflies, Differential Equations Dynam. Systems 2 (1994), 11-18. 


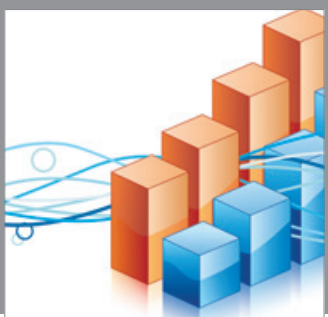

Advances in

Operations Research

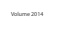

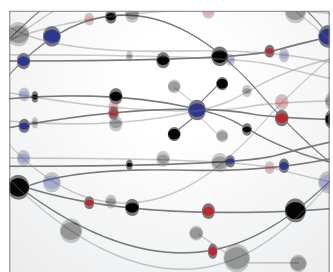

\section{The Scientific} World Journal
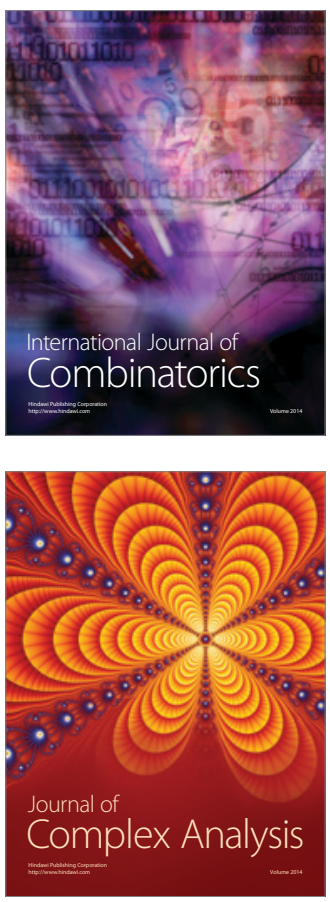

International Journal of

Mathematics and

Mathematical

Sciences
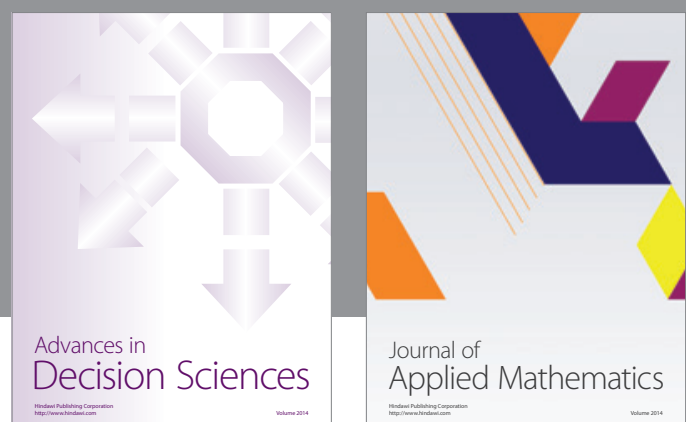

Journal of

Applied Mathematics
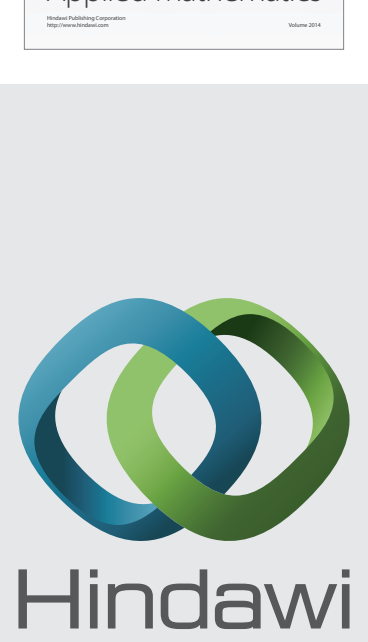

Submit your manuscripts at http://www.hindawi.com
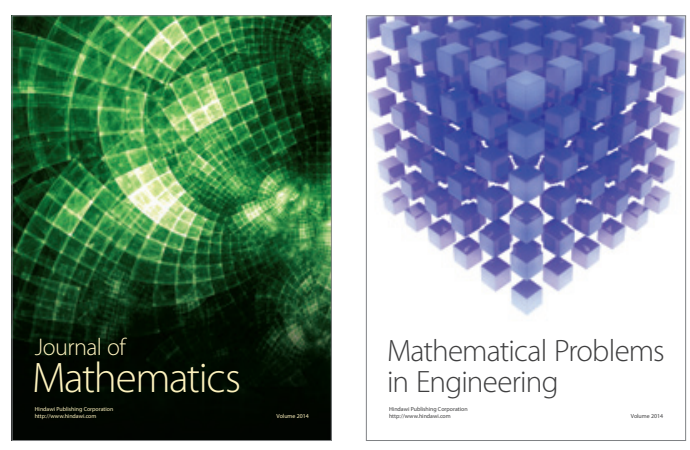

Mathematical Problems in Engineering
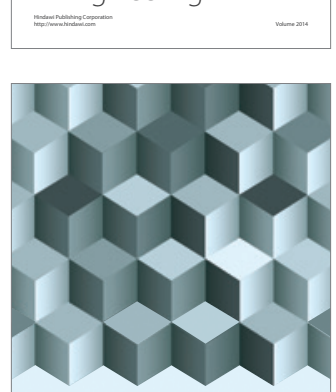

Journal of

Function Spaces
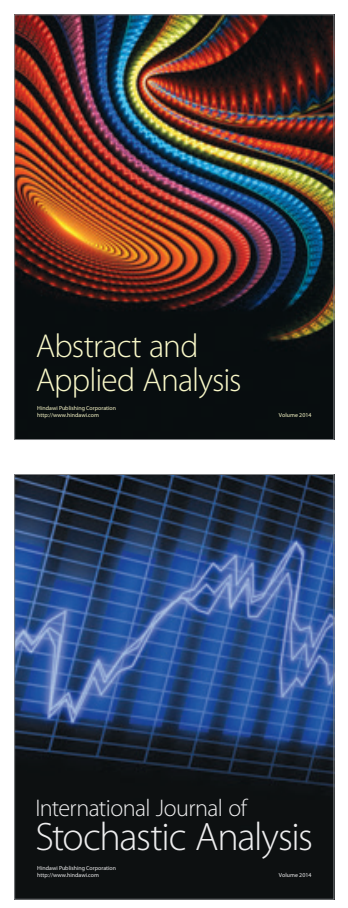

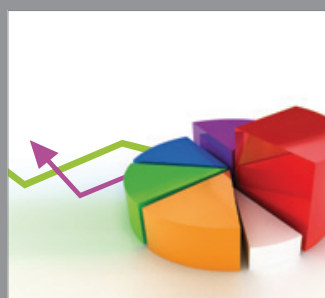

ournal of

Probability and Statistics

Promensencen
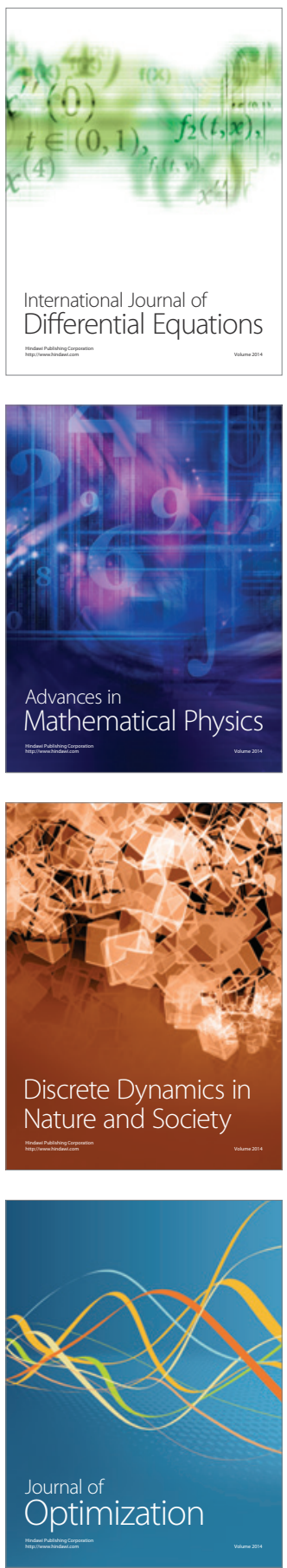